\title{
Measurement-based Evaluation of Cooperative Awareness for V2V and V2I Communication
}

\author{
Mate Boban and Pedro M. d'Orey \\ NEC Laboratories Europe, NEC Europe Ltd. \\ \{mate.boban,pedro.dorey\}@neclab.eu
}

\begin{abstract}
We analyze the efficacy of cooperative awareness enabled by periodic message exchange between vehicles and roadside infrastructure. To measure cooperative awareness, we use three metrics: 1) neighborhood awareness ratio; 2) ratio of neighbors above range; and 3 ) packet delivery rate. Using the measurement data collected within the scope of the DRIVE-C2X project in four European test sites, we analyze the efficacy of cooperative awareness in urban, suburban, and highway environments. Further, we investigate the ability of periodic message exchange to enable cooperative awareness for both Vehicle to Vehicle (V2V) and Vehicle to Infrastructure (V2I) links and with different effective transmit power levels. Our results show that: i) the efficacy of cooperative awareness varies greatly in different environments; ii) V2V and V2I communication have distinct awareness and interference patterns; and iii) high awareness levels imply high interference; therefore, a balance needs to be found between them, depending on the context that the vehicles are in.
\end{abstract}

Keywords-Cooperative Awareness, Empirical Evaluation, Vehicular Networks, Intelligent Transportation Systems, Interference.

\section{INTRODUCTION}

Cooperative awareness is the basis for most safety Intelligent Transportation Systems (ITS) applications proposed by standardization bodies [1]. Using the information provided by cooperative messaging, vehicles and Road Side Units (RSUs) are able to create a map of their surroundings, which is then used as input for safety applications that detect potentially hazardous situations. To enable cooperative awareness, standardization bodies have proposed specific messages for that purpose: in the European Union (EU), Cooperative Awareness Messages (CAMs) have been specified as part of the standard [2], whereas in the United States (U.S.), the same functionality is enabled by the Basic Safety Message (BSM) [3], [4]. These messages are exchanged periodically and contain location, speed, and direction of the vehicle, among other information.

To gain insight into the efficacy of cooperative awareness, we analyze the following metrics: 1) Neighborhood Awareness Ratio (NAR): ratio of neighbors within a given range from which a message has been received within time $t$;2) Ratio of Neighbors Above Range (RNAR): ratio of neighbors outside a given range to all neighbors from which a message has been received within time $t$; and 3) Packet Delivery Ratio (PDR). Since the purpose of cooperative message exchange is timely notification of vehicles and infrastructure about existence of other vehicles, the NAR metric measures the proportion of vehicles in a given Region Of Interest (ROI) that receive

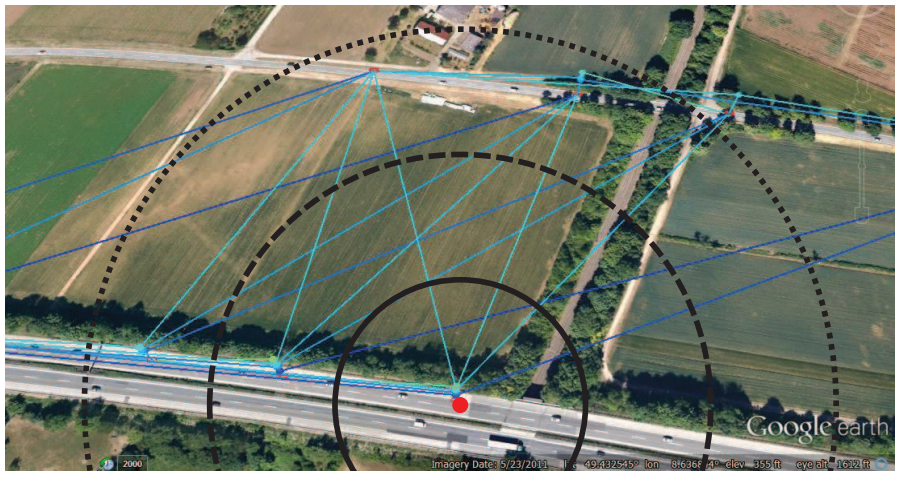

Fig. 1. Neighborhood awareness is based on the broadcast of periodic messages and allows gathering relevant information on the evolving neighborhood within a given awareness zone (e.g., black concentric circles). However, increasing awareness can also result in interference from distant vehicles (vehicles outside the designated awareness zone), whose information might be less relevant than that of the nearby vehicles.

at least one message from the transmitting vehicle in time interval $t$ (and are thus aware of the transmitting vehicle). Conversely, the more distant the transmitting vehicle is, the less relevant the messages from that vehicle are for majority of safety applications. To that end, the RNAR metric measures the proportion of vehicles outside the ROI, from which the messages are received. In future scenarios, where a high percentage of vehicles will be equipped with the communication equipment, high RNAR would imply high interference, and thus low overall system throughput. Therefore, in terms of the communications performance, a well-functioning transmit system would aim to increase NAR, while at the same time keeping RNAR reasonably low.

We elaborate on our previous study of cooperative awareness [5] by: i) separating the data into different type of environments (qualitatively classified into highway, suburban, and urban; ii) including V2I communication analysis (apart from $\mathrm{V} 2 \mathrm{~V}$ ); iii) analyzing the impact of variable transmit power on awareness; and iv) analyzing the data from two additional test sites (Italy and Finland).

The rest of the paper is organized as follows. Section II discusses the related work. Section III describes the DRIVE $\mathrm{C} 2 \mathrm{X}$ communications platform, the selected metrics, and the environments where measurements were made. Section IV shows the results of the measurement data analysis in terms of delivery rate, awareness and interference performance. Section $\mathrm{V}$ concludes the paper. 


\section{RELATED WORK}

Extensive research has been conducted to study cooperative awareness in Vehicular Ad Hoc Networks (VANETs), with most studies resorting to analytical models or simulations. While previous work has mainly focused on the assessment of communication performance, fewer studies looked at the cooperative awareness level provided to applications. In addition, the vast majority of previous studies have focused solely on the evaluation of Vehicle to Vehicle (V2V) performance of periodic beaconing.

With respect to assessment of communication performance in Vehicular Networks using simulations, Mittag et al. [6] compared single and multi-hop broadcast performance. They concluded that limited benefit is achieved when using multihop communication instead of single-hop for cooperative awareness. Van Eenennaam et al. [7] verified analytically that the three main dimensions that make the solution space of beaconing in VANETs are transmission power, generation rate and message duration, and showed how different beaconing configurations support Cooperative Adaptive Cruise Control (CACC). Noori et al. [8] performed simulations to study the probability of beacon delivery in an urban scenario and showed how packet delivery is impacted by increasing vehicle density and different road types. Kloiber et al. [9] analyzed the ability of cooperative message exchange to inform the vehicles about hazardous situations under challenging Medium Access Control (MAC) conditions. Several studies (e.g., [10], [11]) proposed improving awareness levels or reducing the channel load in VANETs by adaptive modification of beacon transmission power or generation rate.

Regarding empirical evaluation of communication and application performance in VANETs, Martelli et al. [12] analyzed the Packet Inter-Reception time (PIR). Their results showed that PIR follows a power-law distribution (i.e., long-lasting outages occur with certain periodicity). Furthermore, PIR is strongly affected by Line of Sight (LOS) conditions, with up to five-fold performance drop in case of LOS obstruction by vehicles. Bai et al. [13] performed an extensive study on the impact of controllable parameters (transmit power, modulation scheme) and uncontrollable factors (distance, environment, velocity) on the performance of IEEE 802.11p [14] radios in terms of PDR. In a similar study, Santa et al. [15] analyzed the influence of several parameters on the performance of CAMs using an experimental testbed and showed that the LOS conditions, equipment installation point and hardware capabilities are key variables in the network performance. Boban et al. [16] demonstrated the importance of accurate channel model selection for correctly simulating the application-level performance in terms of throughput, packet delivery, and latency.

Apart from analyzing the conventional communication performance (e.g., throughput, delay), several studies proposed using information-centric metrics (e.g., awareness quality [6], [17], update delay [18], and PIR [11]). For instance, Kloiber et al. [18] proposed the Update Delay metric, which is defined for a pair of vehicles as the time interval between the expected CAM reception and the actual message reception. These metrics allow for a better understanding of the impact of the underlying vehicular communication system on applicationlevel performance.

\section{EXPERIMENTAL EVALUATION OF COMMUNICATION PERFORMANCE}

This section defines formally the PDR, NAR and RNAR metrics, and describes the DRIVE C2X experimental vehicle and roadside platform and the measurement test sites environments and related system settings.

\section{A. Metrics}

Apart from Packet Delivery Ratio (PDR), a well established metric in evaluation of communication systems, we use two metrics we introduced in our previous paper [5] to measure the efficacy of cooperative awareness: Neighborhood Awareness Ratio (NAR) and Ratio of Neighbors Above Range (RNAR). Below, we formally define all three metrics.

1) Packet Delivery Ratio (PDR): the ratio of the number of correctly received packets to the number of transmitted packets. Formally, for a vehicle $i$, the PDR is calculated as $P D R_{i}=\frac{P R_{i}}{P T_{i}}$, where $P R_{i}$ is the number of packets sent by $i$ that were correctly received by the surrounding vehicles and $P T_{i}$ is the total number of packets sent by $i$ during a given experiment. We measure PDR during the entire experiment duration, i.e. the time interval $t$ over which PDR is measured equals the experiment duration. This metric provides the indication of the link quality and effective and maximum communication range. Effective communication range is defined as the maximum distance below which the PDR is above a given threshold (e.g., 0.9), whereas maximum communication range is the distance above which the PDR is equal to 0 .

2) Neighborhood Awareness Ratio (NAR): the proportion of vehicles in a specific range from which a message was received in a defined time interval. Formally, for vehicle $i$, range $r$, and time interval $t, N A R_{i, r, t}=\frac{N D_{i, r, t}}{N T_{i, r, t}}$, where $N D_{i, r, t}$ is the number of vehicles within $r$ around $i$ from which $i$ received a message in $t$ and $N T_{i, r, t}$ is the total number of vehicles within $r$ around $i$ in $t$ (we use $t=1$ second). This metric measures the efficacy of cooperative awareness messaging.

3) Ratio of Neighbors Above Range $(R N A R)^{1}$ : for a vehicle $i$, range $r$, and time interval $t$, the ratio of neighbors that are above a certain distance from the observed vehicle is defined as $R N A R_{i, r, t}=\frac{N A_{i, r, t}}{N_{i, t}}$, where $N A_{i, r, t}$ is the number of vehicles above $r$ from which $i$ received a message in $t$ (again, we use $t=1$ second) and $N_{i, t}$ is the total number of vehicles from which $i$ received a message in $t$ (irrespective of $r$ ). This metric gives an indication of potentially unnecessary traffic overheard from distant neighbors. Once the technology is deployed at a large scale (i.e., with communication equipment installed in most vehicles), such traffic will translate to unwanted interference.

\footnotetext{
${ }^{1}$ Also referred to as Neighborhood Interference Ratio (NIR) in our previous work [5].
} 
TABLE I. Description of Measurement Test Sites and Parameters

\begin{tabular}{|c|c|c|c|c|}
\hline \multirow[t]{3}{*}{$\begin{array}{l}\text { Location } \\
\text { Scenarios }\end{array}$} & $\begin{array}{c}\text { Gothenburg, Sweden } \\
\text { Suburban } \\
(57.710316,11.94238)\end{array}$ & $\begin{array}{l}\text { Helmond, the Netherlands } \\
\text { Suburban } \\
(51.472803,5.622418)\end{array}$ & $\begin{array}{c}\text { Tampere, Finland } \\
\text { Suburban } \\
(\text { lon }<23.847835, \text { lat }<61.45894)\end{array}$ & Trento, Italy \\
\hline & $\begin{array}{c}\text { Highway } \\
(57.718424,11.918331)\end{array}$ & $\begin{array}{c}\text { Highway } \\
(51.477243,5.620085)\end{array}$ & $\begin{array}{c}\text { Highway } \\
(\text { lat }>61.45894 \text { and lat }<61.491023) \\
(\text { lon }>23.790289 \text { and lon }<23.843118)\end{array}$ & $\begin{array}{c}\text { Highway } \\
(45.934435,11.087010)\end{array}$ \\
\hline & & & $\begin{array}{l}\text { Urban } \\
\text { Otherwise }\end{array}$ & \\
\hline Route Length (Max.) & $11 \mathrm{~km}$ & $5.5 \mathrm{~km}$ & $22 \mathrm{~km}$ & $60 \mathrm{~km}$ \\
\hline Time & $\begin{array}{c}\text { June } 2013 \\
\text { (9 a.m. to } 5 \text { p.m) }\end{array}$ & $\begin{array}{l}\text { September } 2012 \\
(9 \text { a.m. to } 5 \text { p.m. })\end{array}$ & $\begin{array}{l}\text { April and May } 2013 \\
\text { (7 a.m. to } 1 \text { p.m.) }\end{array}$ & $\begin{array}{l}\text { July to October } 2013 \\
\text { (7 a.m. to } 2 \text { p.m.) }\end{array}$ \\
\hline Number of Vehicles & 6 & 7 & 3 & $3 / 4$ \\
\hline Vehicle Type & Personal & Personal & Personal & Personal \\
\hline Antenna Type & Omni-directional & Omni-directional & Omni-directional & Omni-directional \\
\hline Antenna Location & Rooftop & Rooftop & Rooftop & Rooftop \\
\hline Antenna Height & approx. $1.55 \mathrm{~m}$ & approx. $1.44-1.66 \mathrm{~m}$ & approx. $1.5 \mathrm{~m}$ & approx. $1.49 \mathrm{~m}$ \\
\hline Number of RSUs & 0 & 0 & 0 & 5 \\
\hline RSU Antenna & N/A & N/A & N/A & Two Corner Reflector \\
\hline
\end{tabular}

\section{B. Experimental platform}

DRIVE-C2X project designed and evaluated a set of applications enabled by V2V and V2I communication in test sites throughout Europe. The DRIVE-C2X system uses ITS-G5 compliant radios that operate in the $5.9 \mathrm{GHz}$ frequency band. The default value for transmit power was set to $21 \mathrm{dBm}$. On vehicles, whose heights ranged from 1.4 meters to 1.7 meters, omni-directional antennas were placed on the roof. Across test sites, vehicles had different communication system setup, including different radios, cable losses, antenna gains and placements, etc. All of these parameters resulted in significant variations of the effective transmit power output at each vehicle - this is in line with what is expected in the production-grade systems once the communication devices are installed in the cars due to different system designs across manufacturers. The radios transmit CAMs that are in line with the ETSI standard [19]. CAMs contain node information (e.g., position, speed, and sensor information) and are broadcast to one-hop neighbors over the control channel. Positioning information was provided by GPS receivers on the vehicles. In the analyzed datasets, CAMs were sent at $10 \mathrm{~Hz}$ frequency and had the size of 100 Bytes.

\section{Measurement test sites}

The empirical evaluation of cooperative awareness in VANETs presented in this paper is based on analysis of logging information. All nodes (vehicles and RSUs) record all received and transmitted messages during the several test runs. In all test sites, vehicles were driven in normal traffic conditions with the presence of other vehicle types and respecting traffic rules.

In test sites in Sweden and Finland, combined with antenna gains and cable losses, the effective vehicle transmit power ranged between 10 and $20 \mathrm{dBm}$. In Trento, Italy, there were 5 RSUs with the antenna placed at heights between 9 and $11 \mathrm{~m}$ at the positions and locations indicated in Table II. One RSU is installed on a highway on an overhead gantry $11 \mathrm{~m}$ above the road surface. It is equipped with two corner reflector antennas each having $14 \mathrm{dBi}$ nominal gain, beam width 30 degrees in azimuth and 60 degrees in elevation. Remaining RSUs are installed next to the highway at the height of $9 \mathrm{~m}$. Both vehicles and RSUs have a nominal output power of $21 \mathrm{dBm}$. Combined with antenna gains and cable and insertion losses, this yields
TABLE II. TEST Site ItAly RSU Locations

\begin{tabular}{lccc}
\hline Id & Position (lat, lon) & Height & Installed \\
\hline 251 & $45.909728,11.03248$ & $9 \mathrm{~m}$ & Pole \\
252 & $45.905776,11.02953$ & $11 \mathrm{~m}$ & Overhead Gantry \\
253 & $45.86776,11.005438$ & $9 \mathrm{~m}$ & Pole \\
254 & $45.871724,11.007555$ & $9 \mathrm{~m}$ & Pole \\
255 & $45.8569,11.000692$ & $9 \mathrm{~m}$ & Overhead Gantry
\end{tabular}

$27 \mathrm{dBm}$ transmit power on the vehicles, and $32 \mathrm{dBm}$ on RSUs. These power settings are markedly higher than in remaining test sites, where the transmit power on vehicles was between 10 and $20 \mathrm{dBm}$. In the Netherlands, the vehicles used for testing were a combination of vehicles used in the other test sites.

In the test sites Sweden, the Netherlands and Finland, no logging data was available for RSUs, therefore the results contain $\mathrm{V} 2 \mathrm{~V}$ communication tests only. In Italy, on the other hand, logging data was available for both V2V and V2I communication. More details on the experimental setup are given in Table III.

\section{RESUlTS}

In this section, we present and discuss the results of collected during the DRIVE-C2X measurement campaign in terms of PDR, NAR, and RNAR. The results are aggregated per vehicle (over messages transmission) and per test site (over different vehicles) for different environments (urban, suburban, and highway). Each distance bin is 25 meters for PDR and 50 meters for NAR and RNAR, with the plotted data point centered in the middle of the distance bin. Error bars represent one standard deviation around the mean of the measured variable for each vehicle. For statistical relevance, we consider solely bins with at least 40 data points. With respect to NAR and RNAR, for all results and plots shown in the following, one second window was used for determining the reception of messages from direct neighbors.

\section{A. Packet Delivery Rate}

Figs. 2, 3, 4, and 5 show the Packet Delivery Ratio (PDR) as a function of distance for $\mathrm{V} 2 \mathrm{~V}$ and V2I communications for different measurement locations. 


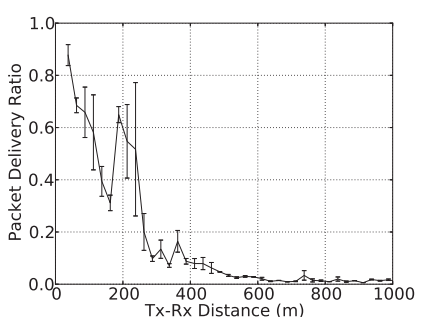

(a) Overall V2V results - Highway.

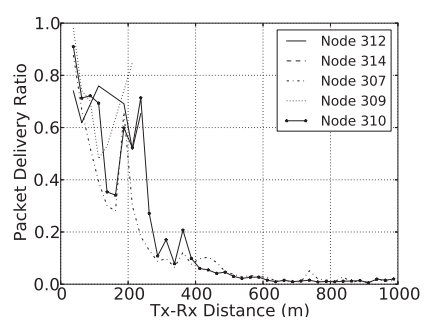

(b) Per-vehicle V2V results - Highway.

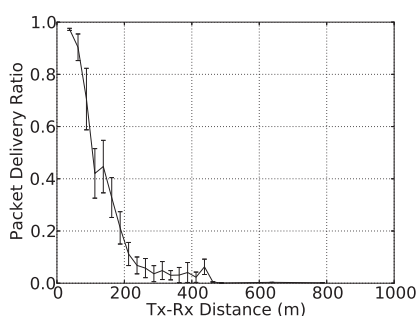

(c) Overall V2V results - Suburban.

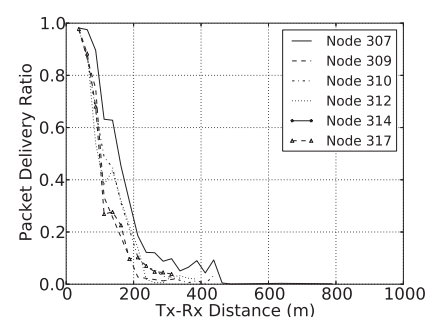

(d) Per-vehicle V2V results - Suburban.

Fig. 2. Packet Delivery Ratio (PDR) for Test Site Sweden.

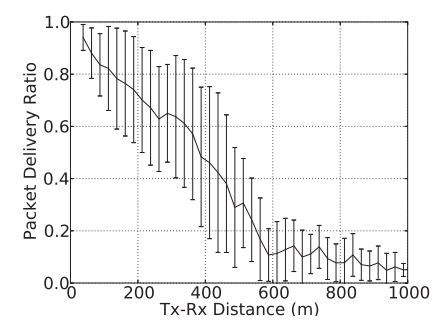

(a) Overall V2V results - Highway.

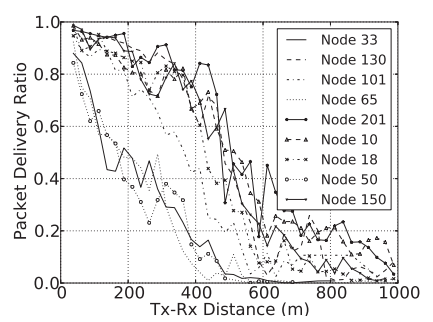

(b) Per-vehicle V2V results - Highway.

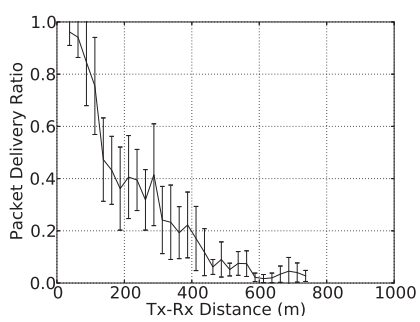

(c) Overall V2V results - Suburban.

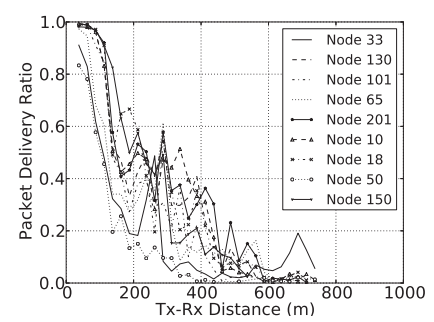

(d) Per-vehicle V2V results - Suburban.

Fig. 3. Packet Delivery Ratio (PDR) for Test Site the Netherlands.

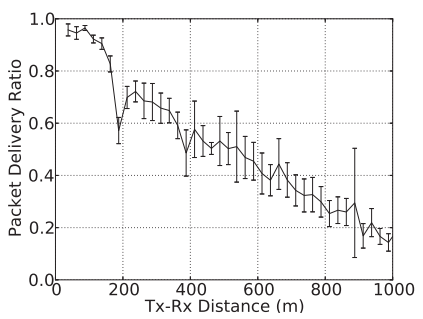

(a) Overall V2V results.

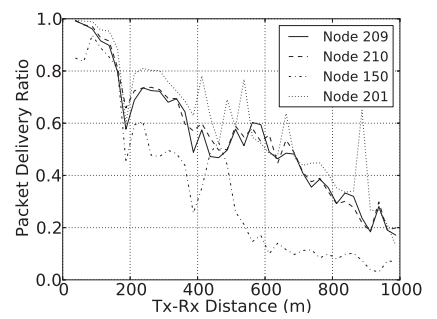

(b) Per-vehicle V2V results

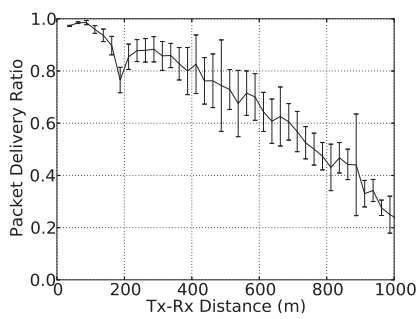

(c) Overall V2I results

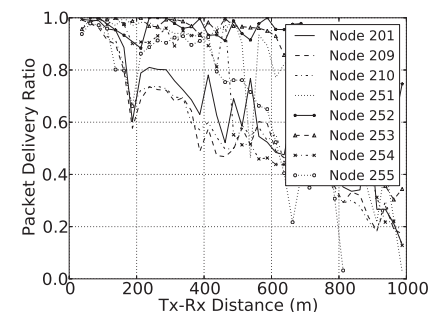

(d) Per-vehicle V2I results

Fig. 4. Packet Delivery Ratio (PDR) for Test Site Italy (Highway).

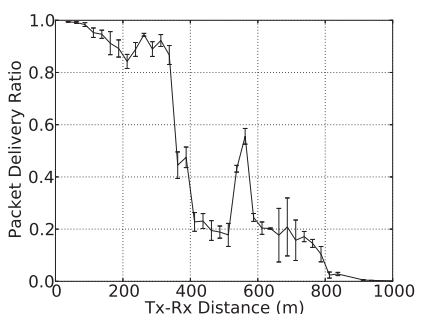

(a) Overall V2V results - Highway

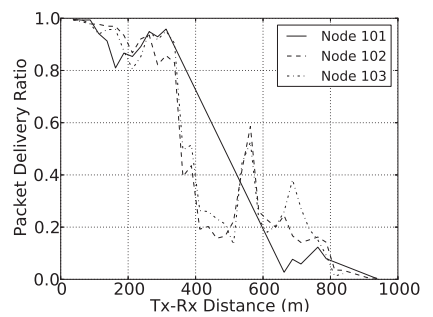

(b) Per-vehicle V2V results - Highway

Fig. 5. V2V Packet Delivery Ratio (PDR) for Test Site Finland.

1) $V 2 V:$ As expected, for all test sites, the PDR decreases as the node separation increases, although some fluctuations can be observed in the presented results. PDR (and, by consequence, the effective and maximum communication range) varies greatly between test sites and between qualitatively classified propagation environments. When considering the environment type, the communication ranges are increasing in the following order for a given test site: urban, suburban, and highway (see, e.g., Fig. 3 for difference between highway and suburban PDR). The harsher propagation environment present in (sub)urban scenarios, including frequent non-LOS conditions due to surrounding objects (e.g., other vehicles, buildings, and trees), affects considerably the link quality and

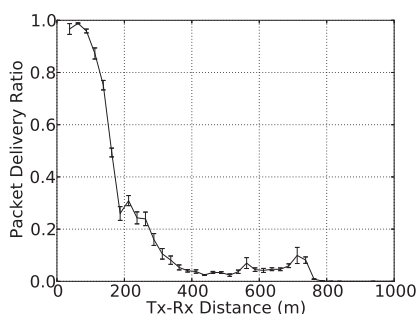

(c) Overall V2V results - Urban

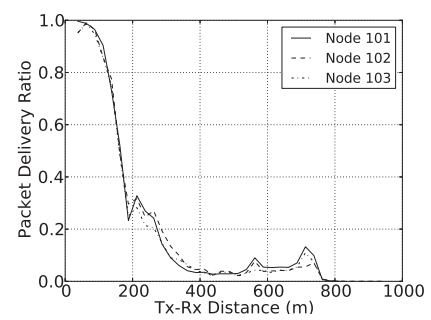

(d) Per-vehicle V2V results - Urban

consequently the successful packet delivery. This is in line with previous measurements studies (e.g.,[20], [21]).

However, the results for the same qualitatively classified environment may vary substantially from one test site to another. This is most evident for the highway scenario where the maximum communication range varies from approximately $600 \mathrm{~m}$ in Sweden (Fig. 2(a)) and Finland (Fig. 5(a)) to more than $1000 \mathrm{~m}$ in Italy (Fig. 4(a)). Therefore, while the qualitative classification of environments is useful as a rough guide for separating different propagation regions in a single location, it is not advisable to use such classification alone when trying to apply results from one measurement location 
to the other, even if they are of similar type (e.g., abovemenitoned highways).

In addition, the difference between communication system setup in vehicles (e.g. different radios, cable losses and antenna gains and placements) impact the communication performance. For example, one of the main reasons for the improved performance in the Italian test site (Fig. 4(a)) is the higher effective transmit power of vehicles.

For a specific measurement location, the non-monotonic decrease of PDR over distance is mainly due to: i) in case of LOS communication, the dominating two-ray ground reflection model [16]; and ii) in case of non-LOS communication, variations in LOS obstruction level (e.g., deep or slight nonLOS due to a building at intersection). Our results in terms of PDR are in line with the analytic results obtained by An et al. [17] and the empirical results by Visintainer et al. [22] for the highway scenario.

2) V2I: The V2I PDR results are presented in Fig. 4(c) and Fig. 4(d) for the Italian test site (highway scenario). These results are in line with the study by Paier et al. [23], with the increased PDR in case of our measurements due to higher transmit powers (32 dBm EIRP on RSUs and $27 \mathrm{dBm}$ on vehicles, compared to $15.5 \mathrm{dBm}$ in Paier et al.).

Compared to V2V results in the same location (Fig. 4(a)), V2I PDR is significantly higher due to two main reasons: 1) advantageous position of RSUs (9-11 $\mathrm{m}$ above ground), giving the RSUs unobstructed LOS at larger distances; and 2) the increased effective transmission power of RSUs.

\section{B. Neighborhood Awareness Ratio}

1) $V 2 V:$ As evidenced in our previous work [5], there is a clear relation between PDR and neighborhood awareness, as both metrics are strongly affected by the link quality. Across test sites, the relationship between different environments and NAR is quite clear: the more complex the environment, the lower the NAR at a given distance. The most clear comparison can be seen on test site Finland (Table III and Fig. 9): in urban environment, $90 \%$ NAR can be achieved at a maximum of $200 \mathrm{~m}$, compared to $350 \mathrm{~m}$ and $400 \mathrm{~m}$ in suburban and highway environments, respectively. Furthermore, looking more deeply at Figs. 6, 7, 8, and 9, we can see that qualitative separation of environments into urban, suburban, and highway cannot be generalized across test sites; for example, NAR in Sweden on highway (Fig. 6(a)) is considerably different than in the same environment in the Netherlands (Fig. 7(a)) and Italy (Fig. 8(a)). This is in line with the PDR results discussed in Section IV-A. Therefore, a protocol that is able to dynamically adjust to the current environment would be useful for adapting the power of transmitted CAMs. When analyzing the pervehicle neighborhood results, we can observe that, for a given distance bin, the performance fluctuations between different vehicles is pronounced in all scenarios. This is the result of both the environment changes over small distance as well as different system setup on vehicles (e.g., antenna placement, cable loss, etc.).

2) V2I: While among the analyzed environments only one contains V2I communication (Italy), it is clear that the advantageous positions and antenna characteristics (higher
TABLE III. Distance Above Which Neighborhood Awareness RATIO (NAR) FALLS BELOW 90\%

\begin{tabular}{ccccc}
\hline Environment & Sweden & The Netherlands & Finland & Italy \\
\hline Highway V2V & $100 \mathrm{~m}$ & $250 \mathrm{~m}$ & $400 \mathrm{~m}$ & $200 \mathrm{~m}$ \\
Suburban V2V & $100 \mathrm{~m}$ & $150 \mathrm{~m}$ & $350 \mathrm{~m}$ & N/A \\
Urban V2V & N/A & N/A & $200 \mathrm{~m}$ & N/A \\
Highway V2I & N/A & N/A & N/A & $650 \mathrm{~m}$
\end{tabular}

gain) of RSUs create a better propagation environment, thus resulting in NAR that is above $90 \%$ up to $700+\mathrm{m}$ (Fig. 8(c)). Note that Figs. 8(c) and 8(d) contain communication from vehicles to RSUs, not the other way around, which would result in even higher NAR due to higher transmit antenna gains.

\section{Ratio of Neighbors Above Range (RNAR)}

1) $V 2 V$ : Figs. 10, 11, 12, and 13 show the Ratio of Neighbors Above Range (RNAR) for different test sites. In case of $\mathrm{V} 2 \mathrm{~V}$ communication, RNAR exhibits approximately exponential behavior, with progressively fewer vehicles detected at higher distances (e.g., proportion of vehicles above 400 meters mostly contained within 10\%). For safety applications requiring information from immediate neighborhood, such behavior is beneficial, since it implies that most periodic messages that a vehicle receives are useful. While the trend of RNAR is similar across the environments, due to different surroundings and transmit power settings, even environments qualitatively characterized as the same have significantly different RNAR values (e.g., at $400 \mathrm{~m}$ on a highway in Sweden it is close to $0 \%$ (Fig. 10(a)), whereas it is above $20 \%$ on highway in Finland (Fig. 13(a)).

2) V2I: Figs. 12(c) and 12(d) show a stark contrast in V2I RNAR compared to the V2V scenarios. Whereas in V2V scenarios, the RNAR tapers off after at most $500 \mathrm{~m}$, the large effective range of RSUs results in a large number of far-away vehicles (e.g., more than half of detected vehicles were farther than $500 \mathrm{~m}$ away in Fig. 12(c)). This difference arises due to a very large range of RSUs, which is enabled by their advantageous positions on tall gantries and their higher-gain antennas.

\section{Discussion}

Results in this paper improve the understanding of achievable awareness using periodic V2V and V2I message exchange, along with the resulting impact on possible unwanted interference. Specifically, our results show that V2V links with low effective transmit power can suffer from low neighborhood awareness, particularly in built-up urban areas; at the same time, V2I links can exhibit high awareness rates even above $1 \mathrm{~km}$. On one hand, it is questionable if the neighborhood awareness information is relevant at distances above those required by safety-critical applications. High awareness is closely related to the potentially high interference, which reduces the frequency reuse and negatively impacts the throughput of future vehicular networks. On the other hand, within distances relevant for safety applications, there is a need for as high awareness as possible. 


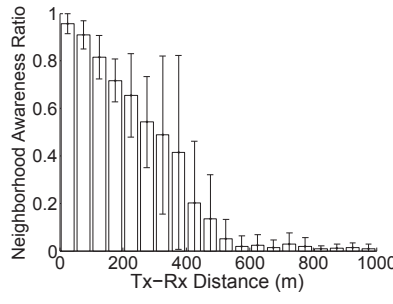

(a) Overall results - Highway.

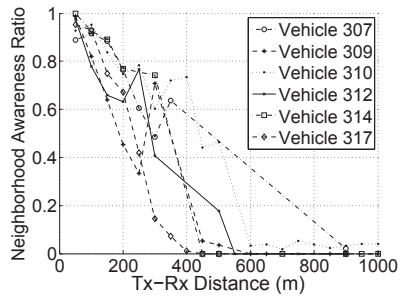

(b) Per-vehicle results - Highway.

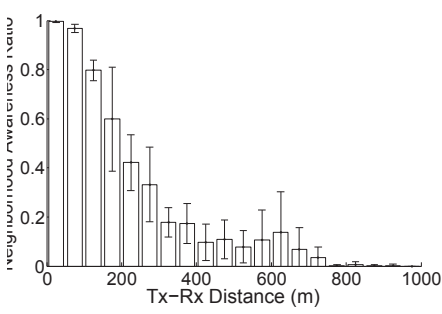

(c) Overall results - Suburban.

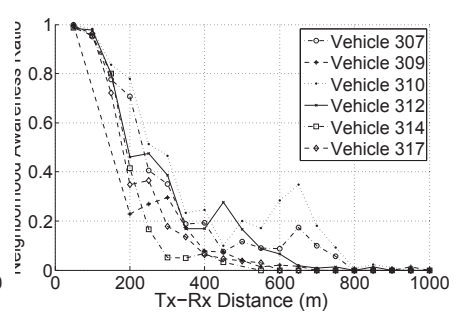

(d) Per-vehicle results - Suburban.

Fig. 6. Neighborhood Awareness Ratio (NAR) for Test Site Sweden.

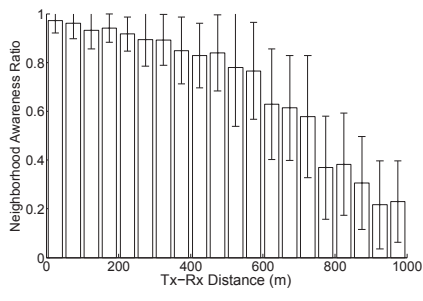

(a) Overall results - Highway.

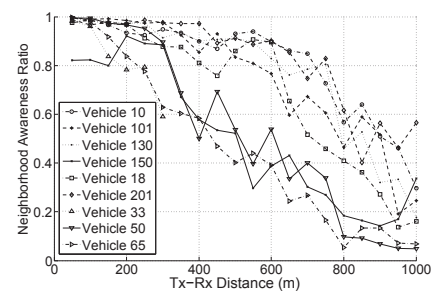

(b) Per-vehicle results - Highway.

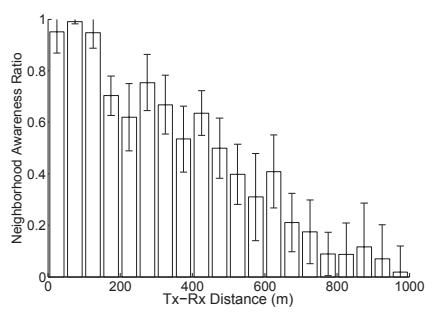

(c) Overall results - Suburban.

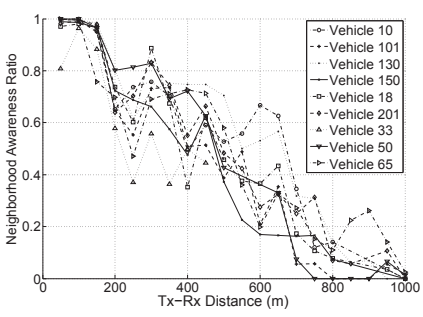

(d) Per-vehicle results - Suburban.

Fig. 7. Neighborhood Awareness Ratio (NAR) for Test Site the Netherlands.

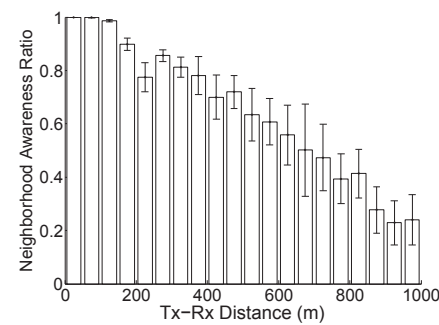

(a) Overall V2V results

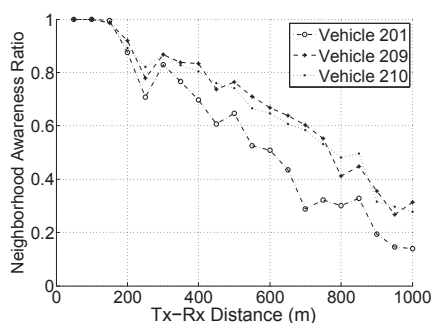

(b) Per-vehicle V2V results.

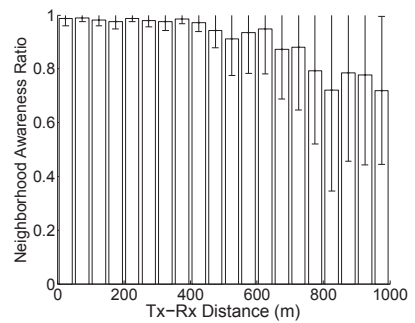

(c) Overall V2I results.

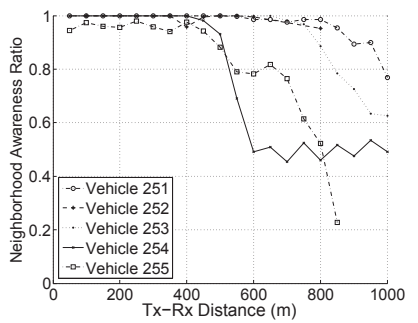

(d) Per-vehicle V2I results.

Fig. 8. Neighborhood Awareness Ratio (NAR) for the Test Site Italy (Highway).

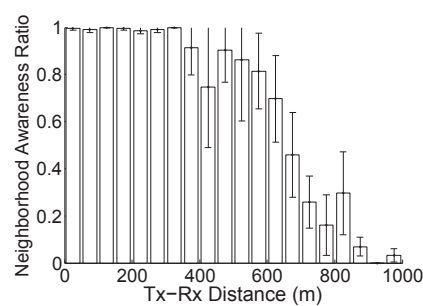

(a) Overall V2V results - Highway.

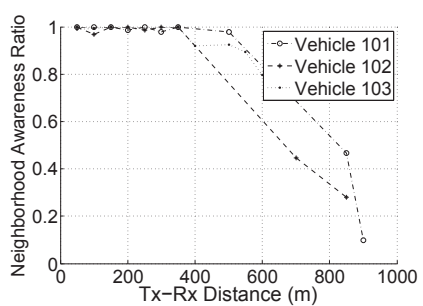

(b) Per-vehicle V2V results - Highway.

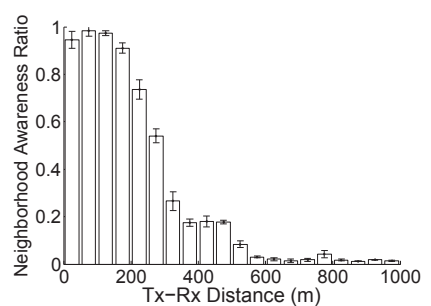

(c) Overall V2V results - Urban.

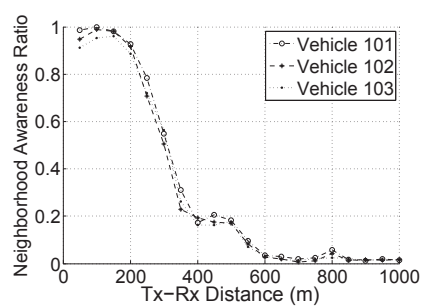

(d) Per-vehicle V2V results - Urban.

Fig. 9. Neighborhood Awareness Ratio (NAR) for Test Site Finland.

\section{Conclusions}

Periodic broadcast of single-hop CAMs is the basis for many safety or traffic efficiency applications, which make use of received information to determine the dynamics of the surroundings. In this study, we empirically assessed cooperative awareness in vehicular networks using large-scale experiments performed in four test sites in Europe within the scope of DRIVE-C2X project. The large-scale datasets comprised of three distinct environments (urban, suburban and highways), two link types (V2V and V2I) and varying effective transmit powers, which allowed us to study the performance under varying conditions.
The presented results demonstrate that cooperative awareness is strongly dependent on link quality and propagation conditions. The harshness of the propagation environment where vehicles move determines the maximum achievable communication range and neighborhood awareness: the more complex the environment, the lower the NAR for a given distance. Consequently, for a given test site, the awareness levels are smaller for urban scenarios, intermediate for suburban scenarios and maximum for highway scenarios. With respect to the link type, the results show that the advantageous positions of RSUs improve the awareness levels for V2I communications when compared with $\mathrm{V} 2 \mathrm{~V}$ communications. Furthermore, higher effective transmit power can, while in- 


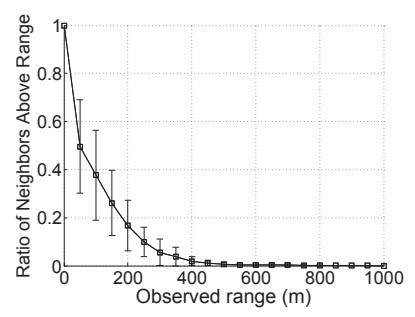

(a) Overall results - Highway.

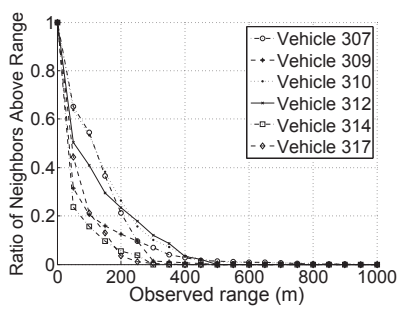

(b) Per-vehicle results - Highway.

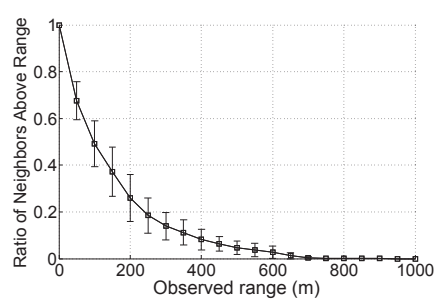

(c) Overall results - Suburban.

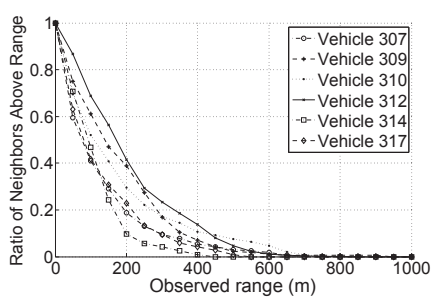

(d) Per-vehicle results - Suburban.

Fig. 10. Ratio of Neighbors Above Range (RNAR) for Test Site Sweden.

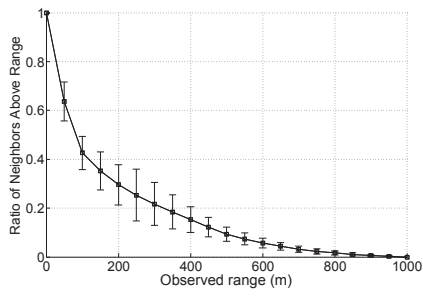

(a) Overall results - Highway.

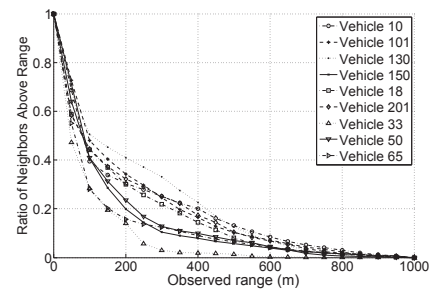

(b) Per-vehicle results - Highway.

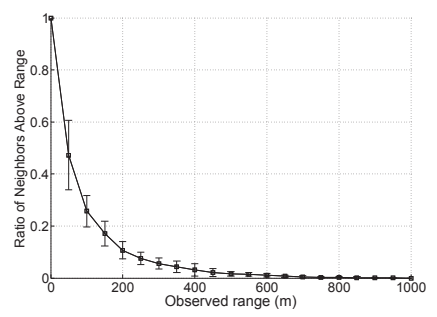

(c) Overall results - Suburban.

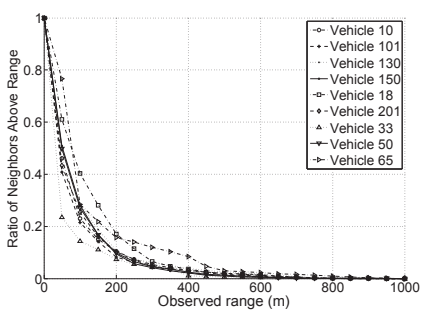

(d) Per-vehicle results - Suburban.

Fig. 11. Ratio of Neighbors Above Range (RNAR) for Test Site the Netherlands.

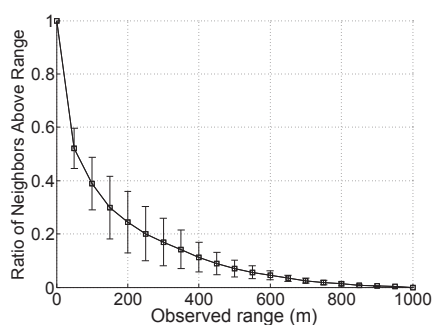

(a) Overall V2V results.

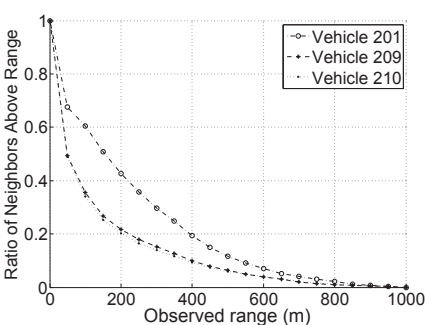

(b) Per-vehicle V2V results.

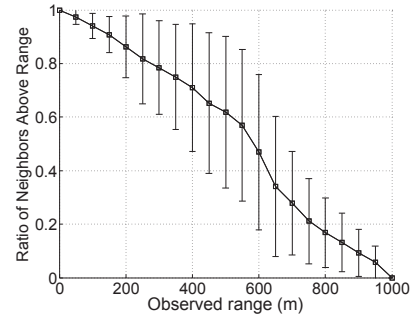

(c) Overall V2I results.

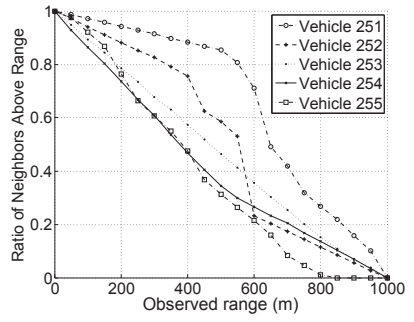

(d) Per-vehicle V2I results.

Fig. 12. Ratio of Neighbors Above Range (RNAR) for Test Site Italy (Highway).

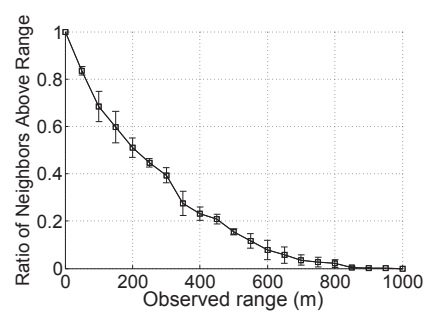

(a) Overall V2V results - Highway.

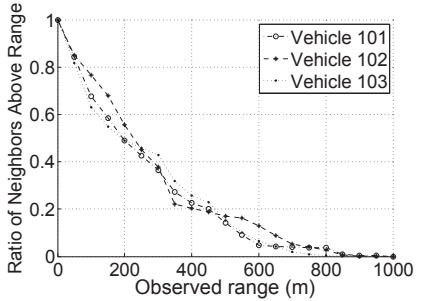

(b) Per-vehicle V2V results - Highway.

Fig. 13. Ratio of Neighbors Above Range (RNAR) for Test Site Finland.

creasing awareness levels, also (prohibitively) increase the interference by far-away nodes; this effect is especially evident for highway scenarios and V2I communication.

With regards to the application performance, our results show that applications requiring high awareness levels of up to $100 \mathrm{~m}$ can be satisfied in virtually all environments. For larger distances, high awareness is available in certain types of environments (e.g., highway). Finally, any application requiring high awareness rates over $300 \mathrm{~m}$ in all environments will need to use either higher transmit power or multi-hop forwarding, at the expense of increasing the interference in its surrounding.

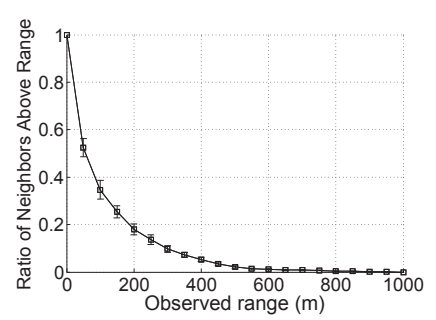

(c) Overall V2V results - Urban.

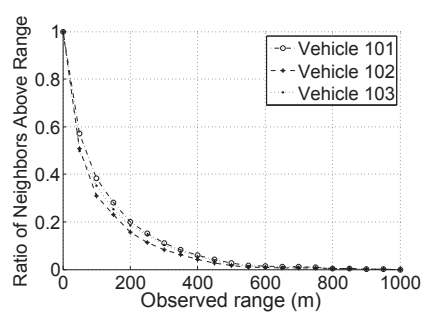

(d) Per-vehicle V2V results - Urban.

As future work, we plan to perform a simulation study to investigate the awareness and interference rates on a largescale (i.e., involving thousands of communicating vehicles), using a realistic propagation model [24] to simulate different environments.

\section{ACKNOWLEDGMENTS}

The research leading to these results has received funding from the European Union's Seventh Framework Programme (FP7/2007-2013) under grant agreement n. 270410, DRIVE $\mathrm{C} 2 \mathrm{X}$. 


\section{REFERENCES}

[1] ETSI TC ITS, "Intelligent Transport Systems (ITS); Vehicular Communications; Basic Set of Applications; Definitions," Tech. Rep. ETSI TR 102638 V1.1.1, June 2009.

[2] _ _ "Intelligent Transport Systems (ITS); Vehicular Communications; Basic Set of Applications; Part 2: Specification of Cooperative Awareness Basic Service," Tech. Rep. EN 302 637-2, 2014.

[3] SAE International, "SAE J2735 Dedicated Short Range Communications (DSRC) Message Set Dictionary," Tech. Rep. J2735_200911, 2009.

[4] J. Kenney, "Dedicated Short-Range Communications (DSRC) Standards in the United States," Proceedings of the IEEE, vol. 99, no. 7, pp. 1162 1182, July 2011.

[5] P. d'Orey and M. Boban, "Empirical Evaluation of Cooperative Awareness in Vehicular Communications," in IEEE 79th Vehicular Technology Conference: VTC2014-Spring, May 2014.

[6] J. Mittag, F. Thomas, J. Härri, and H. Hartenstein, "A Comparison of Single- and Multi-hop Beaconing in VANETs," in ACM International Workshop on VehiculAr InterNETworking, Systems and Applications, 2009, pp. 69-78.

[7] M. van Eenennaam, W. Wolterink, G. Karagiannis, and G. Heijenk, "Exploring the Solution Space of Beaconing in VANETs," in IEEE Vehicular Networking Conference, 2009, pp. 1-8.

[8] H. Noori and B. B. Olyaei, "A Novel Study on Beaconing for VANETbased Vehicle to Vehicle Communication: Probability of Beacon Delivery in Realistic Large-Scale Urban Area using 802.11p," in International Conference on Smart Communications in Network Technologies, 2013.

[9] B. Kloiber, T. Strang, M. Rockl, and F. de Ponte-Muller, "Performance of CAM based safety applications using ITS-G5A MAC in high dense scenarios," in IEEE Intelligent Vehicles Symposium (IV). IEEE, 2011, pp. $654-660$

[10] B. Kloiber, J. Harri, and T. Strang, "Dice the TX power - Improving Awareness Quality in VANETs by Random Transmit Power Selection," in IEEE Vehicular Networking Conference (VNC), Nov 2012, pp. 5663.

[11] T. Tielert, D. Jiang, H. Hartenstein, and L. Delgrossi, "Joint Power/Rate Congestion Control Optimizing Packet Reception in Vehicle Safety Communications," in ACM international Workshop on Vehicular internetworking, Systems, and Applications, 2013, pp. 51-60.

[12] F. Martelli, M. Elena Renda, G. Resta, and P. Santi, "A Measurementbased Study of Beaconing Performance in IEEE 802.11p Vehicular Networks," in IEEE International Conference on Computer Communications, 2012, pp. 1503-1511.
[13] F. Bai, D. D. Stancil, and H. Krishnan, "Toward Understanding Characteristics of Dedicated Short Range Communications (DSRC) from a Perspective of Vehicular Network Engineers," in International Conference on Mobile Computing and Networking, 2010, pp. 329-340.

[14] “IEEE Draft Standard IEEE P802.11p/D9.0,” Tech. Rep., July 2009.

[15] J. Santa, F. Pereniguez, A. Moragon, and A. Skarmeta, "Experimental Evaluation of CAM and DENM Messaging Services in Vehicular Communications," Transportation Research Part C: Emerging Technologies, vol. 46, pp. 98 - 120, 2014.

[16] M. Boban, W. Viriyasitavat, and O. K. Tonguz, "Modeling Vehicle-tovehicle Line of Sight Channels and its Impact on Application-layer Performance," in ACM International Workshop on Vehicular Internetworking, Systems and Applications, 2013, pp. 91-94.

[17] N. An, T. Gaugel, and H. Hartenstein, "VANET: Is 95\% Probability of Packet Reception safe?" in International Conference on ITS Telecommunications, 2011, pp. 113-119.

[18] B. Kloiber, C. Garcia, J. Härri, and T. Strang, "Update Delay: A New Information-centric Metric for a Combined Communication and Application Level Reliability Evaluation of CAM based Safety Applications," in ITS World Congress, 2012.

[19] ETSI TC ITS, "Intelligent Transport Systems (ITS); Vehicular Communications; Basic Set of Applications; Part 2: Specification of Cooperative Awareness Basic Service," Tech. Rep. TS 102 637-2 V1.2.1, 2011.

[20] M. Boban, T. T. V. Vinhoza, J. Barros, M. Ferreira, and O. K. Tonguz, "Impact of Vehicles as Obstacles in Vehicular Ad Hoc Networks," IEEE J. Select. Areas Commun., vol. 29, no. 1, pp. 15-28, January 2011.

[21] R. Meireles, M. Boban, P. Steenkiste, O. Tonguz, and J. Barros, "Experimental study on the impact of vehicular obstructions in vanets," in IEEE Vehicular Networking Conference (VNC), Dec. 2010, pp. 338 -345 .

[22] F. Visintainer, L. DOrazio, M. Darin, and L. Altomare, "Cooperative Systems in Motorway Environment: The Example of Trento Test Site in Italy," in Advanced Microsystems for Automotive Applications 2013, ser. Lecture Notes in Mobility. Springer, 2013, pp. 147-158.

[23] A. Paier, R. Tresch, A. Alonso, D. Smely, P. Meckel, Y. Zhou, and N. Czink, "Average downstream performance of measured ieee $802.11 \mathrm{p}$ infrastructure-to-vehicle links," in 2010 IEEE International Conference on Communications (ICC) Workshops, May 2010, pp. 1 -5.

[24] M. Boban, J. Barros, and O. Tonguz, "Geometry-based vehicle-tovehicle channel modeling for large-scale simulation," IEEE Transactions on Vehicular Technology, vol. PP, no. 99, pp. 1-1, 2014. 